ARTICLE

\title{
van der Waals driven anharmonic melting of the 3D charge density wave in $\mathrm{VSe}_{2}$
}

\author{
Josu Diego ${ }^{1}$, A. H. Said ${ }^{2}$, S. K. Mahatha ${ }^{3}$, Raffaello Bianco', Lorenzo Monacelli ${ }^{4,5}$, Matteo Calandra ${ }^{5,6,7}$, \\ Francesco Mauri (1D) ${ }^{4,5}$, K. Rossnagel (i) ${ }^{3,8}$, Ion Errea (1) ${ }^{1,9,10 凶}$ \& S. Blanco-Canosa (1) ${ }^{10,11 凶}$
}

Understanding of charge-density wave (CDW) phases is a main challenge in condensed matter due to their presence in high-Tc superconductors or transition metal dichalcogenides (TMDs). Among TMDs, the origin of the CDW in $\mathrm{VSe}_{2}$ remains highly debated. Here, by means of inelastic $x$-ray scattering and first-principles calculations, we show that the CDW transition is driven by the collapse at $110 \mathrm{~K}$ of an acoustic mode at $\mathbf{q}_{C D W}=\left(\begin{array}{l}2.250 \\ 0\end{array}\right.$ 0.7) r.l.u. The softening starts below $225 \mathrm{~K}$ and expands over a wide region of the Brillouin zone, identifying the electron-phonon interaction as the driving force of the CDW. This is supported by our calculations that determine a large momentum-dependence of the electron-phonon matrix-elements that peak at the CDW wave vector. Our first-principles anharmonic calculations reproduce the temperature dependence of the soft mode and the $T_{C D W}$ onset only when considering the out-of-plane van der Waals interactions, which reveal crucial for the melting of the CDW phase.

\footnotetext{
${ }^{1}$ Centro de Física de Materiales (CSIC-UPV/EHU), 20018 San Sebastián, Spain. ${ }^{2}$ Advanced Photon Source, Argonne National Laboratory, Lemont, IL 60439, USA. ${ }^{3}$ Ruprecht Haensel Laboratory, Deutsches Elektronen-Synchrotron DESY, 22607 Hamburg, Germany. ${ }^{4}$ Dipartimento di Fisica, Università di Roma La Sapienza, Roma, Italy. ${ }^{5}$ Graphene Labs, Fondazione Instituto Italiano di Tecnologia, Genoa, Italy. ${ }^{6}$ Dipartimento di Fisica, Università di Trento, Via Sommarive 14, 38123 Povo, Italy. ${ }^{7}$ Sorbonne Universités, CNRS, Institut des Nanosciences de Paris, UMR7588, F-75252 Paris, France. ${ }^{8}$ Institut für Experimentelle und Angewandte Physik, Christian-Albrechts-Universität zu Kiel, 24098 Kiel, Germany. ${ }^{9}$ Fisika Aplikatua 1 Saila, Gipuzkoako Ingeniaritza Eskola, University of the Basque Country (UPV/EHU), San Sebastián, Spain. ${ }^{10}$ Donostia International Physics Center (DIPC), 20018 San Sebastián, Spain. ${ }^{11}$ IKERBASQUE, Basque

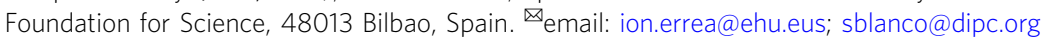


T he study of electronic ordering and charge-density-wave (CDW) formation is attracting massive efforts in condensed matter physics ${ }^{1}$. In particular, its dynamical nature is the focus of a strong debate in correlated oxides and high- $T \mathrm{c}$ superconducting cuprates ${ }^{2}$, where fluctuations of the charge order parameter $^{3}$, dispersive CDW excitations ${ }^{4}$, and phonon anomalies ${ }^{5}$ are observed. Microscopically, the subtle balance between electron-phonon interaction (EPI) and nested portions of the Fermi surface (singularities in the electronic dielectric function, $\chi_{\mathrm{q}}$, at $\mathbf{q}_{\mathrm{CDW}}=2 k_{\mathrm{F}}$ ) determines the origin and stabilization of the charge periodicities ${ }^{6}$. While the Fermi surface nesting scenario survives for $1 \mathrm{D}$ and quasi-1D systems (Peierls transition), its role in higher dimensions remains largely questioned ${ }^{7,8}$.

Among the solids showing electronic charge ordering, layered transition metal dichalcogenides (TMDs) represent the first crystalline structures where $3 \mathrm{D}$ CDWs were discovered ${ }^{9} .1 T-\mathrm{VSe}_{2}$ (space group $P \overline{3} \mathrm{~m} 1)$ belongs to the series of layered TMDs that develops a $3 \mathrm{D}-\mathrm{CDW}$ as a function of temperature, $T_{\mathrm{CDW}}=110 \mathrm{~K}$. However, unlike the isostructural $1 T$-TiSe 2 , which adopts a commensurate $2 \times$ $2 \times 2 \mathrm{CDW}$ ordering with $\mathbf{q}_{\mathrm{CDW}}=\left(\begin{array}{lll}0.5 & 0 & 0.5\end{array}\right)$ r.l. $\mathrm{u}^{10}, 1 T-\mathrm{VSe}_{2}$ develops a more complex temperature dependence 3D incommensurate pattern in its CDW phase with a $\mathbf{q}_{\mathrm{CDW}}=(0.250-0.3)$ r.l.u $\mathrm{CDW}$ wave vector ${ }^{11}$, modulating the interlayer distances. $1 T-\mathrm{VSe}_{2}$ is rather unique among the $1 T$-polytypes because it develops anomalies in its transport properties and magnetic susceptibility 12 that more closely resemble those of $2 \mathrm{H}$-polytypes $\left(T_{\mathrm{CDW}}\left[2 \mathrm{H}-\mathrm{NbSe}_{2}\right]=\right.$ $33 \mathrm{~K}, T_{\mathrm{CDW}}\left[2 \mathrm{H}-\mathrm{TaSe}_{2}\right]=122 \mathrm{~K}$ ) and presents the lowest onset temperature among them, i.e., $T_{\mathrm{CDW}}\left[1 T-\mathrm{TiSe}_{2}\right]=200 \mathrm{~K}, T_{\mathrm{CDW}}[1 T$ $\left.\mathrm{TaS}_{2}\right]=550 \mathrm{~K}^{11}$. The sizable difference between $T_{\mathrm{CDW}}\left[1 \mathrm{~T}-\mathrm{VSe}_{2}\right]$ and its $1 T$ counterparts can be attributed to the occurrence of large fluctuation effects that lower the mean-field transition temperature ${ }^{13}$ or to the out-of-plane coupling ${ }^{14}$ between neighboring $\mathrm{VSe}_{2}$ layers assisted by the weak short-range van der Waals interactions ${ }^{15}$. Moreover, the theoretical input based on ab initio calculations is also limited for all these TMDs undergoing CDW transitions due to the breakdown of the standard harmonic approximation for phonons, which cannot explain the stability of the high-temperature undistorted phases ${ }^{16}$. This hinders the study of both the origin and the melting of the electronically modulated state, complicating the comprehensive understanding of the CDW formation.

From the electronic point of view, angle-resolved photoemission (ARPES) experiments in $\mathrm{VSe}_{2}$ reported asymmetric dogbone electron pockets centered at $M(L)^{17}$ that follow the threefold symmetry of the Brillouin zone (BZ) interior, with nesting vectors closely matching those observed by $\mathrm{x}$-ray scattering ${ }^{18}$. The formation of the $\mathrm{CDW}$ results from the 3D warping of the Fermi surface in the $M L$ plane (Fig. 1a shows the high-symmetry points of the Brillouin zone of the hexagonal lattice of $\mathrm{VSe}_{2}$ ). Moreover, photoemission data also find a partial suppression of the density of states near $E_{\mathrm{F}}$ on the nested portion below $180 \mathrm{~K}$, indicating that a pseudogap opens at the Fermi surface ${ }^{19}$. However, a detailed investigation of the electronic structure is complicated by the $3 \mathrm{D}$ nature of the $\mathrm{CDW}$ order, and the momentum dependence of the EPI and the response of the lattice to the opening of the gap at $E_{\mathrm{F}}$ remains unsolved. In fact, inelastic $\mathrm{x}$-ray scattering (IXS) and theoretical calculations discarded the Fermi surface nesting scenario proposed for $2 \mathrm{H}$ $\mathrm{NbSe}_{2}{ }^{20,21}$ and $1 T-\mathrm{TiSe}_{2}{ }^{22,23}$ and emphasized the critical role of the momentum dependence of the EPI. In addition, it has been recently demonstrated that large anharmonic effects are required to suppress the CDW phases in TMDs and understand their phase diagrams, both in the bulk and in the monolayer limit ${ }^{16,24-27}$. Indeed, an evolution from the $(4 \times 4) \mathrm{CDW}$ in bulk $\mathrm{VSe}_{2}$ to a $(\sqrt{7} \times \sqrt{3})$ electronic reconstruction has been reported by means of scanning tunneling microscopy ${ }^{28}$, imperatively calling for a comprehensive description of the nature of the $3 \mathrm{D} \mathrm{CDW}$ in $\mathrm{VSe}_{2}$.

\section{Results}

Quasi-elastic central peak. Figure $1 \mathrm{~b}$ displays the temperature dependence of the elastic signal at the critical wave vector $\mathbf{q}_{\mathrm{CDW}}=\left(\begin{array}{lll}2.25 & 0 & 0.7\end{array}\right)$ r.l.u upon cooling from $300 \mathrm{~K}$. The elastic line due to incoherent scattering is barely visible at high temperature and is temperature independent down to $150 \mathrm{~K}$, implying low structural disorder. Below $150 \mathrm{~K}$ (Supplementary Fig. 4), a smooth increase of the quasi-elastic intensity is observed at $\mathbf{q}=\mathbf{q}_{\mathrm{CDW}}$ due to low-energy critical fluctuations and displays a sharp onset at the CDW transition $T \approx 110 \mathrm{~K}$. No indications of charge instabilities were observed along the $\Gamma \rightarrow M$ and $\Gamma \rightarrow L$ directions. The mean-field critical exponent obtained in the disordered phase at $T>T_{\mathrm{CDW}}, \gamma=1.303 \pm 0.004$, is consistent with the existence of a $3 \mathrm{D}$ regime of critical fluctuations of an order parameter of dimensions $n=2$, as expected for a classical $X Y$ universality class $^{29}$. A similar critical exponent has been observed in the quasi-1D conductor blue bronze $\mathrm{K}_{0.3} \mathrm{MoO}_{3}{ }^{30}$ and $\mathrm{ZrTe}_{3}{ }^{31}$, which develops a giant Kohn anomaly at the CDW transition.

Experimental and theoretical phonons. Figure 1c displays the momentum dependence of the inelastic spectra at $(2+h 00.7)$ r.l.u. for $0.15<h<0.45$ at $300 \mathrm{~K}$. Optical phonons appear above $17 \mathrm{meV}$ and do not overlap with the acoustic branches. At all momentum transfers, $0<h<0.5$, the spectrum consists of 2 phonons, labeled as $\omega_{1}$ and $\omega_{2}$ in Fig. 1d, in good agreement with the results of the theoretical calculations (see Supplementary Fig. 5 for a precise description and assignment of the 2 branches). The third acoustic mode is silent in IXS as its polarization vector is perpendicular to the wave vector. Both $\omega_{1}$ and $\omega_{2}$ belong to the same irreducible representation and, thus, do not cross. For $h<0.2, \omega_{1}$ develops more spectral weight than $\omega_{2}$ and, for $h>0.2$, the intensity of $\omega_{2}$ increases and $\omega_{1}$ leads an apparent asymmetric broadening of $\omega_{2}$, as depicted in Fig. 1d. To obtain quantitative information of the frequency and the phonon lifetime, the experimental scans were fitted using standard damped harmonic oscillator functions convoluted with the experimental resolution of $\approx 1.5 \mathrm{meV}$ (see Fig. $1 \mathrm{~d}$ and Supplementary Fig. 6 for a detailed analysis of the fitting). The frequencies of the low-energy acoustic branches $\omega_{1}$ and $\omega_{2}$ start around 4 and $8 \mathrm{meV}$, respectively, and end at $\approx 13 \mathrm{meV}$. Remarkably, the results of our ab initio anharmonic phonon calculations with the stochastic self-consistent harmonic approximation (SSCHA $)^{32-34}$, which are performed with forces calculated within density-funcitonal theory (DFT) and including van der Waals interactions, show that both $\omega_{1}$ and $\omega_{2}$ do not follow a sinusoidal dispersion, but develop a dip at $h \approx 0.25$ r.l.u. The theoretical dispersion nicely matches the experimental data from the zone center to the border of the Brillouin zone (BZ), as shown in Fig. 1e. In fact, the results of the harmonic phonon calculations indicate that the high-temperature structure of $1 T-\mathrm{VSe}_{2}$ is unstable towards a CDW transition. It is clear, thus, that anharmonicity stabilizes $1 T-\mathrm{VSe}_{2}$ at high temperatures. On the other hand, the linewidth extracted from the analysis (Fig. 1f, symbols) of the $\omega_{2}$ mode is resolution limited across the whole BZ. Nevertheless, the linewidth of the $\omega_{1}$ branch is no longer resolution limited between $0.2<h<0.3$ r.l.u. and develops an anomalously large broadening of $\approx 4 \mathrm{meV}$ at $h=0.25$ r.l.u. Again, the experimental broadening is well captured by our calculations (dashed lines in Fig. 1f), indicating that the large enhancement of the broadening is mainly due to the EPI even if the anharmonic contribution to the linewidth also peaks at $h=$ 0.25 r.l.u. (Supplementary Fig. 10).

Given the observation of the phonon broadening at room temperature and the good agreement between theory and experiment, we proceed with the analysis of the lattice dynamics at lower temperatures. At $250 \mathrm{~K}$, the phonon with energy $\approx 7 \mathrm{meV}\left(\omega_{2}\right)$ 
(a)

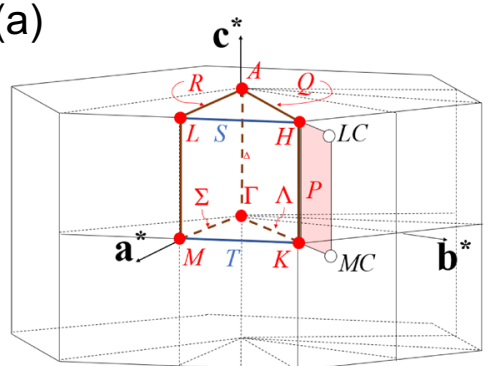

(d)

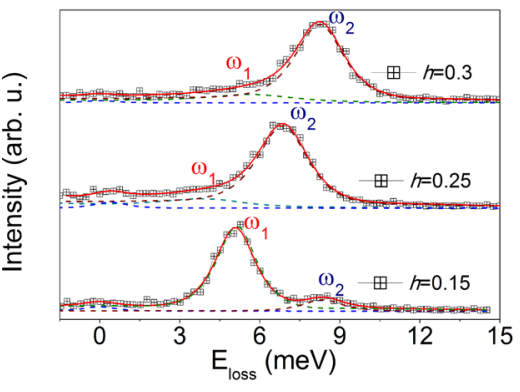

(b)

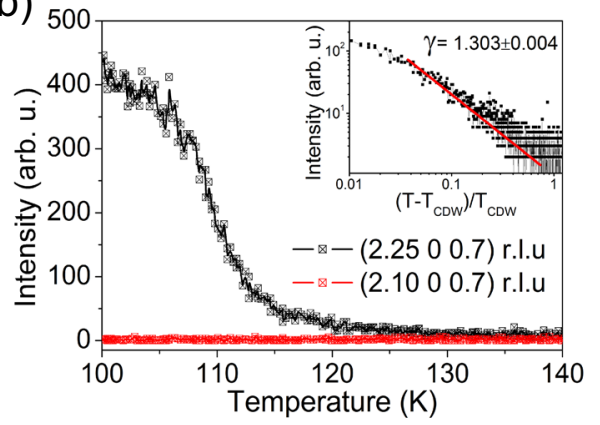

(e)

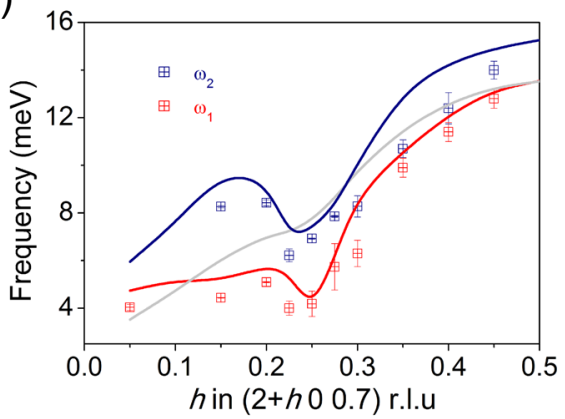

(c)

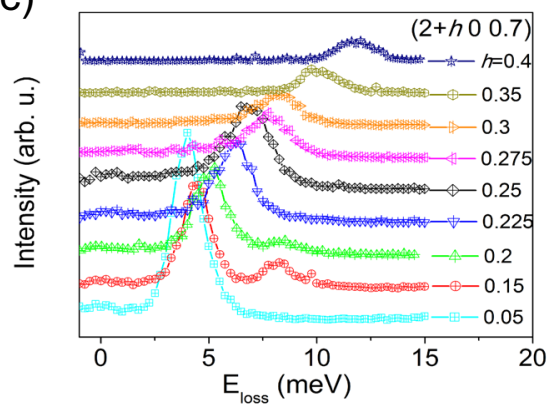

(f)

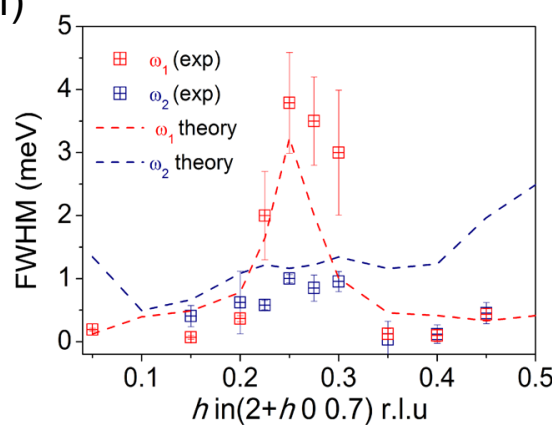

Fig. 1 Elastic peak, CDW, and lattice dynamics at $300 \mathbf{K}$. a The Brillouin zone of space group $P \overline{3} \mathrm{~m} 1$ (164) ${ }^{52}$. b Temperature dependence of the elastic line at (2.25 00.7 ) r.l.u. (reciprocal lattice unit) showing the onset of the CDW at $110 \mathrm{~K}$. Inset, scaling analysis of the elastic peak. c Energy-momentum dependence of the low-energy acoustic phonons at $300 \mathrm{~K}$ from $0.05<h<0.4$ r.l.u. along the $(2+h 00.7)$ direction. The spectra are vertically offset for clarity. d Close-up view of the phonon fitting at $300 \mathrm{~K}$ for selected momentum transfers, identifying the $\omega_{1}$ and $\omega_{2}$ phonons. e Experimental (points) and calculated (solid lines) dispersion of the low-energy acoustic phonons at $300 \mathrm{~K}$. The gray line stands for the silent mode, not observed experimentally. f Momentum dependence of the linewidth for $\omega_{1}$ and $\omega_{2}$ obtained from the fitting of the experimental spectra to damped harmonic oscillators. In panels (e) and (f), the error bars represent the fit uncertainty. The calculated linewidth including the contribution of the electron-phonon interaction and anharmonicity is shown as dashed lines.

shows a clear asymmetric broadening at $\mathbf{q}_{\mathrm{CDW}}$, i.e, the corresponding branch $\omega_{1}$ appears to develop a redshift as a function of temperature (Fig. 2b). The dispersion of $\omega_{2}$ at $150 \mathrm{~K}$ is similar to the one at $300 \mathrm{~K}$. Contrarily, $\omega_{1}$ lowers its energy, softening from room temperature down to $110 \mathrm{~K}$. The softening extends over a wide region of momentum space $0.225<h<0.3$ r.l.u. $\left(0.15 \AA^{-1}\right)$ at $150 \mathrm{~K}$, see green dotted line in Fig. 2a (and Supplementary Fig. 8). The pronounced instability of this acoustic mode and its broad extension in momentum space are consistent with the results of our anharmonic phonon calculations (solid lines in Fig. 2c). The momentum space spread of the softening indicates a substantial localization of the phonon fluctuations in real space due to the EPI, questioning the pure nesting mechanism suggested by ARPES ${ }^{17}$. More importantly, the softening of this branch represents the first indication of the lattice response to the formation of the 3D-CDW in $\mathrm{VSe}_{2}$. The analysis of the linewidth reveals that the lifetime of $\omega_{2}$ remains nearly constant across the $\mathrm{BZ}$ and is resolution limited (Fig. 2d). On the other hand, the softening of the $\omega_{1}$ mode at $150 \mathrm{~K}$ is accompanied by an enhancement of the linewidth, as shown in Fig. $2 \mathrm{~d}$ (6 meV linewidth at $120 \mathrm{~K}$, Fig. $3 \mathrm{f})$ and, again, well modeled by the $\mathrm{ab}$ initio calculations (dashed lines in Fig. $2 \mathrm{~d}$ ).

At the critical temperature, $T_{\mathrm{CDW}}=110 \mathrm{~K}$ and $\mathbf{q} \approx \mathbf{q}_{\mathrm{CDW}}$, the spectrum is dominated by an elastic central peak at zero energy loss $(\mathrm{FWHM}=0.05$ r.l.u. and $\Delta \mathrm{E}=1.6 \mathrm{meV})$, thus, the soft mode is no longer resolvable (see Fig. $3 \mathrm{a}-\mathrm{d}$ and Supplementary Fig. 9). Figure $3 e$ displays the temperature dependence of the soft mode, $\omega_{1}$, as well as the frequency of the phonon obtained ab initio with and without including van der Waals corrections. As plotted in Fig. 3e, the phonon frequency obtained ab initio follows the temperature dependence of the experimental acoustic branch. Moreover, the high temperature $1 T$ structure of $\mathrm{VSe}_{2}$ remains unstable at all temperatures after withdrawing the van der Waals functional from DFT calculation (see blue triangle in Fig. 3e). The softening of the acoustic phonon is accompanied by a linewidth broadening at $T_{\mathrm{CDW}}$ (Fig. $3 \mathrm{f}$ ).

\section{Role of EPI}

Having achieved a comprehensive description of the CDW and its temperature dependence, we address the crucial role of the EPI and nesting mechanism in the formation of the charge modulated state. In Fig. 4, we plot the calculated harmonic phonon frequency together with the electron-phonon linewidth of the three acoustic modes along $\mathbf{q}=\left(\begin{array}{lll}h & 0 & -1 / 3\end{array}\right)$ r.l.u calculated within densityfunctional perturbation theory (DFPT). As it can be seen, the harmonic phonon instability of $\omega_{1}$ coincides with a huge increase of its linewidth associated with the EPI. The softening and the increase of the electron-phonon linewidth specially affect the $\omega_{1}$ mode, which suggests that the electron-phonon matrix elements are strongly mode and momentum dependent and have a strong impact on the real part of the phonon self-energy, which determines the harmonic phonon frequencies ${ }^{8,21}$. This behavior is similar to the one reported for $1 T$-TiSe ${ }_{2}$ and $2 \mathrm{H}-\mathrm{NbSe}_{2}{ }^{20,22}$. The real part of the non-interacting susceptibility $\chi_{0}(\mathbf{q})$, which captures the full Fermi surface topology and also affects the real part of the phonon self-energy (see Supplementary Information), has a softening of around $4 \%$ at $\mathbf{q}_{\mathrm{CDW}}$, which seems insufficient to explain the large softening of the $\omega_{1}$ mode. This suggests that the electron-phonon matrix elements are crucial to induce the harmonic softening and that the topology of the Fermi surface is not the driving mechanism. In order to further clarify the point, we calculate the so-called nesting function $\zeta(\mathbf{q})$ which measures the topology of the Fermi 


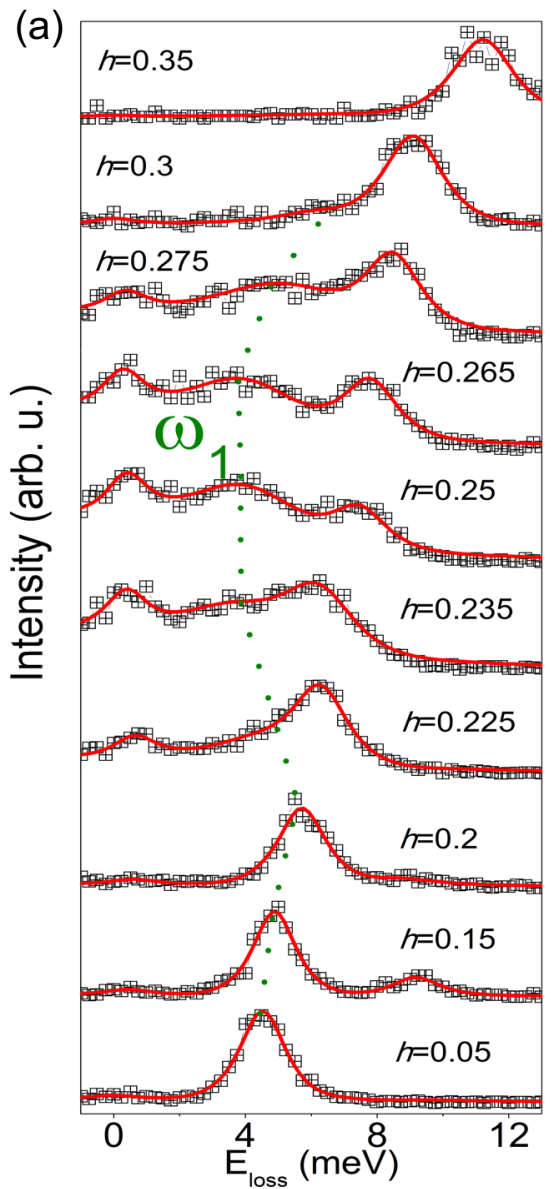

(b)

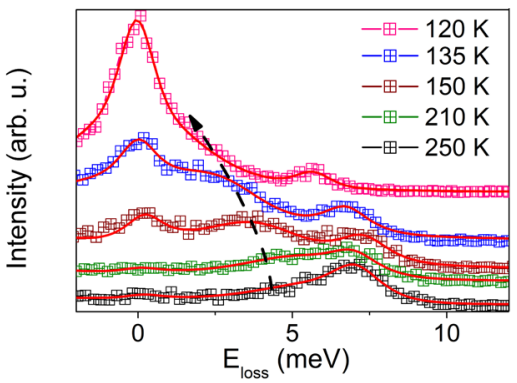

(c)

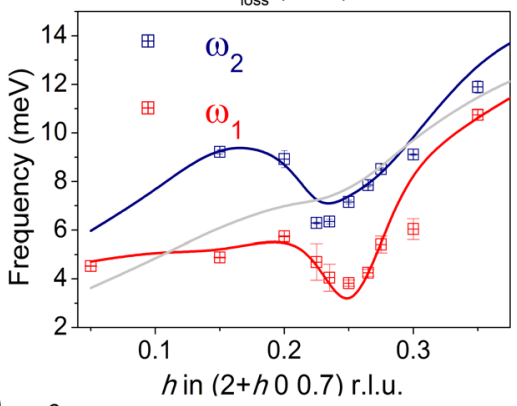

(d)

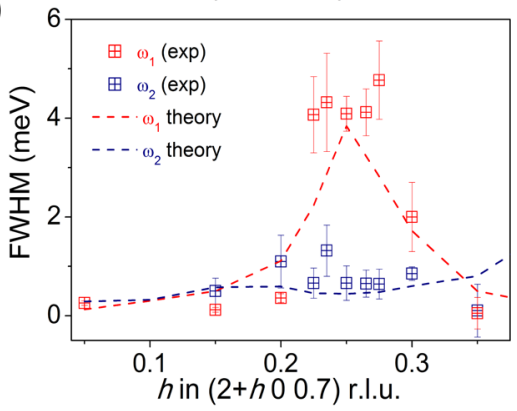

Fig. 2 Lattice dynamics at 150 K. a IXS energy-loss scans at $(2+h 00.7)$ r.l.u for $0.15<h<0.35$ and $150 \mathrm{~K}$. The dotted green line follows the dispersion of the soft phonon mode, $\omega_{1}$ (see text). Each spectrum is vertically shifted for clarity. $\mathbf{b}$ Energy-loss scans as a function of temperature at (2.25 0 0.7) r.l.u. The black arrow follows the softening of the low-energy acoustic mode upon cooling. In both (a) and (b) red lines are the result of the fitting to damped harmonic oscillator functions convoluted with the instrumental resolution. c Momentum dependence of the frequency of the $\omega_{1}$ and $\omega_{2}$ branches at $150 \mathrm{~K}$. The anharmonic phonon dispersions of the acoustic modes obtained at $150 \mathrm{~K}$ are plotted as solid lines. The gray line represents the acoustic mode that is silent in IXS. d Experimental (symbols) and theoretical (dashed lines) momentum dependence of the linewidth for $\omega_{1}$ and $\omega_{2}$. The error bars represent the fit uncertainty. The theoretical calculation accounts for both the electron-phonon and anharmonic contributions to the linewidth.

surface and peaks at the nesting $\mathbf{q}$ points (Supplementary Information). As shown in Fig. 4c, it peaks at $\mathbf{q}_{\mathrm{CDW}}$, which indicates that the CDW vector coincides with a nested region of the Fermi surface. Considering that for constant electron-phonon matrix elements the nesting function coincides with the phonon linewidth given by the EPI, it is illustrative to compare them. Clearly, the phonon linewidth of the $\omega_{1}$ mode coming from the EPI depends much more drastically on momentum than the nesting function: it changes by orders of magnitude as a function of $\mathbf{q}$ while the nesting function only by less than a factor of two. This is highlighted in the ratio between the linewidth and the nesting function plotted in Fig. 4d, which measures the momentum dependence of the electron-phonon matrix elements and should be flat if the electron-phonon matrix elements were constant. This ratio depends much more strongly on momentum than the nesting function itself and resembles the linewidth dependence, underlining again that the momentum dependence of the electron-phonon matrix elements plays a crucial role here. In conclusion, the EPI is the main driving force of the $\mathrm{CDW}$ transition in $1 T-\mathrm{VSe}_{2}$ despite the presence of nesting at $\mathbf{q}_{\mathrm{CDW}}$. Nevertheless, the $\mathbf{q}$-range over which the phonon softens, $\Delta \mathbf{q} \approx 0.075$ r.l.u., even if it coincides with an increase of the electron-phonon linewidth, is a factor of 3 less than in $1 T-\mathrm{TiSe}_{2}{ }^{22}$, where EPI and excitonic correlations are responsible for the structural instability and the CDW order, pointing to an intricate relationship between EPI and Fermi surface nesting scenarios in $\mathrm{VSe}_{2}$.

\section{Discussion}

Our anharmonic calculations, which predict that the $\omega_{1}$ frequency vanishes between 75 and $110 \mathrm{~K}$, are in good agreement with the experimentally measured phonon frequencies and the CDW temperature onset, $T_{\mathrm{CDW}}=110 \mathrm{~K}$. When the SSCHA anharmonic calculation is repeated without including the van der Waals corrections (blue triangles in Fig. 3e), the softest acoustic mode at $\mathbf{q}_{\mathrm{CDW}}$ remains unstable even at room temperature. Remarkably, the weak van der Waals forces (of the order of $\sim 1 \mathrm{mRy} / a_{0}$ for a typical SSCHA supercell calculation) are responsible for the stabilization of the $1 T$ structure of $\mathrm{VSe}_{2}$ and play a crucial role in melting the CDW. On the other hand, the damping ratio, $\Gamma / \tilde{\omega}_{q}$, increases upon cooling and the phonon becomes critically overdamped at $\mathbf{q}_{\mathrm{CDW}}$ and $110 \mathrm{~K}$. The damping ratio $\Gamma / \tilde{\omega}_{q}$ is given by $\omega_{0}=\left(\tilde{\omega}_{q}^{2}-\Gamma^{2}\right)^{1 / 2}$, where $\Gamma$ is the linewidth $\tilde{\omega}_{q}$ is the phonon energy renormalized by the real part of the susceptibility and $\omega_{0}$ is the energy of the phonon fitted to damped harmonic oscillator. The critical exponent derived from the fitting of the phonon frequency $v s$ reduced temperature $\left(\left(\mathrm{T}-\mathrm{T}_{\mathrm{CDW}}\right) /\right.$ $\left.\mathrm{T}_{\mathrm{CDW}}\right), \beta=0.52 \pm 0.04$, agrees with the square-root power law 
(a)

(b)
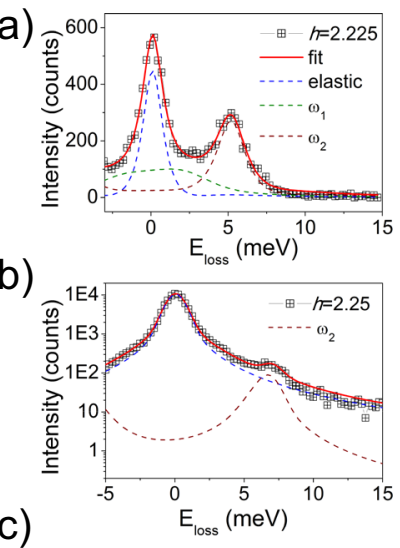

(c)

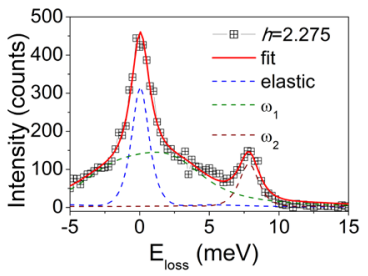

(d)

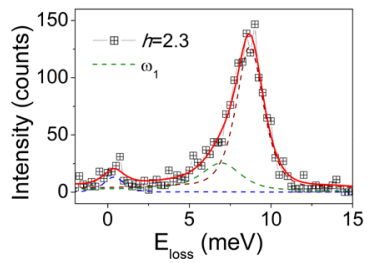

(e)

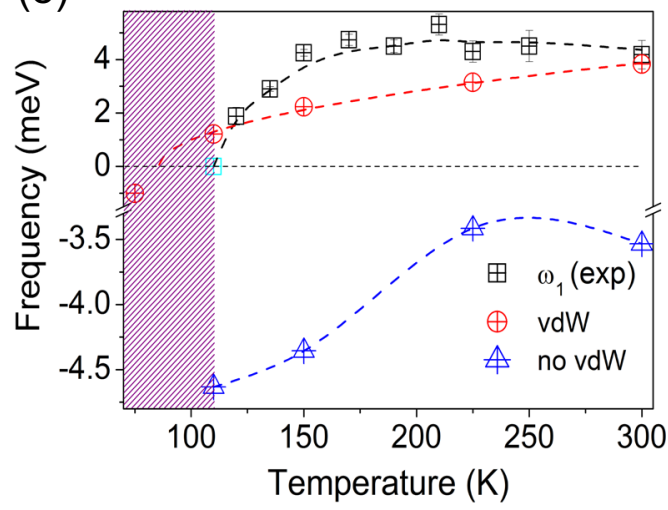

(f)

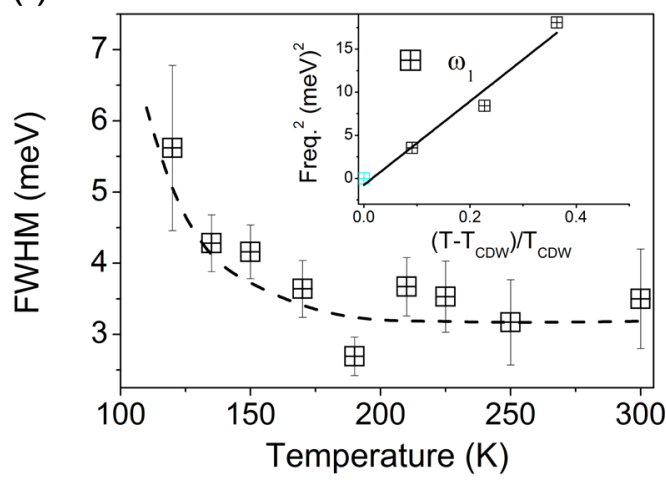

Fig. 3 Phonon collapse and van der Waals melting. a-d Representative IXS spectra at $110 \mathrm{~K}$ and their corresponding fitting. The IXS scan at $h=2.25 \mathrm{r} . \mathrm{l} . \mathrm{u}$. is presented in logarithmic scale due to the large enhancement of the elastic line. $\omega_{1}$ stands for the soft mode. e Temperature dependence of the energy of the $\omega_{1}$ branch and the anharmonic theoretical frequencies obtained with and without van der Waals corrections. The shaded area defines the CDW region. f Temperature dependence of the linewidth. Inset, squared frequency of the soft mode as a function of the reduced temperature. Lines are guides to the eye. The cyan squares in (e) and (f) (inset) refer to the frequency of the $\omega_{1}$ phonon extrapolated to the CDW temperature, since the large enhancement of the elastic line precludes the extraction of its energy from the fitting analysis. The error bars in the experimental data points in panel (e) and (f) represent the fit uncertainty.
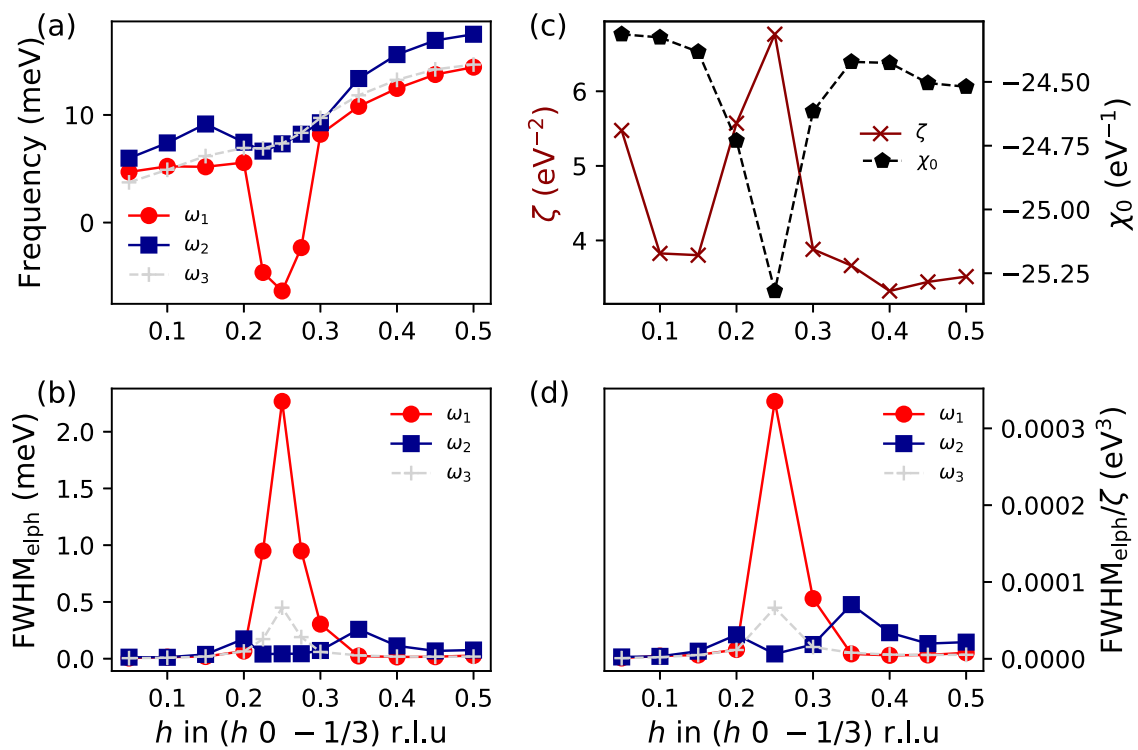

(d)

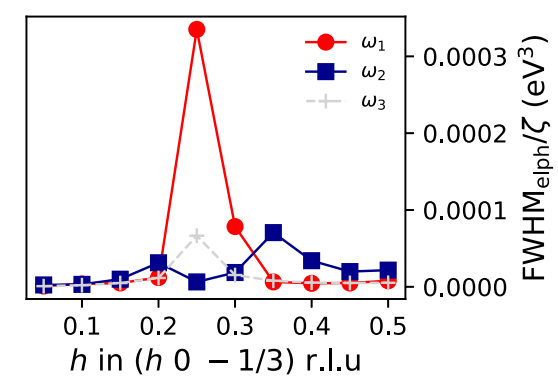

Fig. 4 Electron-phonon interaction vs Fermi surface nesting. a Calculated harmonic phonon spectra of $1 T$-VSe $e_{2}$ along $(h 0-1 / 3)$ r.l.u. Only acoustic modes are shown. The gray line denotes the mode silent in IXS, which is labeled as $\omega_{3}$ here. b Phonon linewidth (full width at half maximum) given by the electron-phonon interaction for the same modes. c Real part of the non-interacting susceptibility, $\chi_{0}$, as well as the nesting function, $\zeta$, at the same wave vectors. d Ratio between the full width at half maximum given by the electron-phonon interaction and the nesting function. 
expected from the mean-field theory (inset of Fig. 3f) and, therefore, fluctuation corrections are unnecessary to invoke the low $T_{\mathrm{CDW}}$ of $\mathrm{VSe}_{2}$ as compared to its $1 T$ counterparts. The critical role of the EPI has been recently suggested by Raman scattering ${ }^{35}$ and DFT calculations ${ }^{36}$. Indeed, revisited ARPES experiments ${ }^{37}$ in $\mathrm{NbSe}_{2}$ revealed a pronounced dispersion along $k_{z}$ discarding the nesting-driven CDW formation and leaving EPI as the major contributor $^{20}$. Although our results indicate an EPI-driven CDW instability, nesting is present and, thus, the charge modulated ground state of $\mathrm{VSe}_{2}$ has to be understood as an interplay between EPI and Fermi surface nesting scenarios.

In conclusion, we have observed with high-resolution IXS that the CDW transition in $1 T-\mathrm{VSe}_{2}$ is driven by the collapse of an

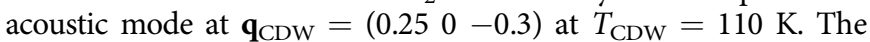
high-temperature $1 T-\mathrm{VSe}_{2}$ phase is stable thanks to anharmonic effects. The observed wide softening in momentum space, the calculated strongly momentum dependent electron-phonon linewidth that peaks at $\mathbf{q}_{\mathrm{CDW}}$, and the weaker dependence on the wave vector of the susceptibility suggest that the EPI is the main driving force of the CDW transition despite the presence of nesting. Moreover, the results show that van der Waals forces are responsible for the melting of the CDW. The dominant role of van der Waals forces here may be attributed to the out-of-plane nature of the CDW, which modulates the interlayer distance. This is not the case in $2 \mathrm{H}-\mathrm{NbSe}_{2}$, where the bulk and monolayer transition temperatures seem to be similar ${ }^{26,38}$. This line of thinking is consistent with the enhancement of the $\mathrm{CDW}$ in monolayer $\mathrm{VSe}_{2}, \mathrm{~T}_{\mathrm{CDW}}=$ $220 \mathrm{~K}^{28}$, since the out-of-plane van der Waals interactions are absent in this case. The critical role of out-of-plane coupling of layers has also been highlighted in the development of the $3 \mathrm{D}$ CDW in high-Tc cuprate superconductors ${ }^{39-41}$.

\section{Methods}

Sample growth and characterization. High-quality single crystals of $\mathrm{VSe}_{2}$ with dimensions $2 \times 2 \times 0.05 \mathrm{~mm}^{3}$ were grown by chemical vapor transport (CVT) using iodine as transport agent (see Supplementary Figs. 1, 2 for their structural, magnetic ${ }^{42}$ and electronic characterization).

\section{Inelastic x-ray scattering (IXS) measurements. The high-resolution IXS} experiments were carried out using the HERIX spectrometer at the 30-ID beamline of the Advanced Photon Source (APS), Argonne National Laboratory. The incident beam energy was $23.72 \mathrm{keV}$ and the energy and momentum resolution was $1.5 \mathrm{meV}$ and $0.7 \mathrm{~nm}^{-1}$, respectively, ${ }^{43}$. The components $(h k l)$ of the scattering vector are expressed in reciprocal lattice units (r.l.u.), $(h k l)=h \mathbf{a}^{*}+k \mathbf{b}^{*}+l \mathbf{c}^{*}$, where $\mathbf{a}^{*}, \mathbf{b}^{*}$, and $c^{*}$ are the reciprocal lattice vectors. The experimental lattice constants of the hexagonal unit cell at room temperature are $a=3.346 \AA, c=6.096 \AA$, and $\gamma=120^{\circ}$. Here, we focus on the low-energy acoustic phonon branches dispersing along the $(0$ $<h<0.50-0.3)$ r.l.u direction in the Brillouin zone near the reciprocal lattice vector $\mathbf{G}_{201}$, thus, in the range $(2+h 0-0.3)$ r.l.u with $0<h<0.5$.

First-principles calculations. The variational SSCHA ${ }^{32-34}$ method was used to calculate temperature-dependent phonons fully accounting for non-perturbative anharmonic effects. The variational free energy minimization of the SSCHA was performed by calculating forces on $4 \times 4 \times 3$ supercells (commensurate with $\mathbf{q}_{\mathrm{CDW}}$ ) making use of DFT within the Perdew-Burke-Ernzerhof $(\mathrm{PBE})^{44}$ parametrization of the exchange-correlation functional. Van der Waals corrections were included within Grimme's semiempirical approach ${ }^{45}$. Harmonic phonon frequencies and electron-phonon matrix elements were calculated within density-functional perturbation theory (DFPT) ${ }^{46}$. The force calculations in supercells needed for the SSCHA as well as the DFPT calculations were performed within the QUANTUM ESPRESSO package ${ }^{47,48}$ (See Supplementary Information for further details on the calculations, which includes citations to refs. ${ }^{49-51}$ ).

\section{Data availability}

The data that support the findings of this study are available from the corresponding author upon reasonable request. See author contributions for specific data sets.

Received: 23 July 2020; Accepted: 22 December 2020;

Published online: 26 January 2021

\section{References}

1. Grüner, G. Charge Density Waves in Solids (Addison-Wesley, 1994).

2. Frano, A., Blanco-Canosa, S., Keimer, B. \& Birgeneau, R. J. Charge ordering in superconducting copper oxides. J. Phys.: Condens. Matter 32, 374005 (2020).

3. Arpaia, R. et al. Dynamical charge density fluctuations pervading the phase diagram of a Cu-based high-Tc superconductor. Science 365, 906-910 (2019).

4. Chaix, $\mathrm{L}$. et al. Dispersive charge density wave excitations in $\mathrm{Bi}_{2} \mathrm{Sr}_{2} \mathrm{CaCu}_{2} \mathrm{O}_{8+\delta}$. Nat. Phys. 13, 952-956 (2017).

5. Le Tacon, $\mathrm{M}$. et al. Inelastic $\mathrm{x}$-ray scattering in $\mathrm{YBa}_{2} \mathrm{Cu}_{3} \mathrm{O}_{6.6}$ reveals giant phonon anomalies and elastic central peak due to charge-density-wave formation. Nat. Phys. 10, 52-58 (2014).

6. Chan, S. K. \& Heine, V. Spin density wave and soft phonon mode from nesting Fermi surfaces. J. Phys. F: Met. Phys. 3, 795-809 (1973).

7. Miao, H. et al. Formation of incommensurate charge density waves in cuprates. Phys. Rev. X 9, 031042 (2019).

8. Johannes, M. D. \& Mazin, I. I. Fermi surface nesting and the origin of charge density waves in metals. Phys. Rev. B 77, 165135 (2008).

9. Wilson, J., Salvo, F. D. \& Mahajan, S. Charge-density waves and superlattices in the metallic layered transition metal dichalcogenides. Adv. Phys. 24, 117-201 (1975).

10. Joe, Y. I. et al. Emergence of charge density wave domain walls above the superconducting dome in 1T-TiSe 2 . Nat. Phys. 10, 421-425 (2014).

11. Rossnagel, K. On the origin of charge-density waves in select layered transition-metal dichalcogenides. J. Phys.: Condens. Matter 23, 213001 (2011).

12. C. F. van Bruggen, C. Magnetic susceptibility and electrical properties of $\mathrm{VSe}_{2}$ single crystals. Solid State Commun. 20, 251-253 (1976).

13. Lee, P. A., Rice, T. M. \& Anderson, P. W. Fluctuation effects at a Peierls transition. Phys. Rev. Lett. 31, 462-465 (1973).

14. Driza, N. et al. Long-range transfer of electron-phonon coupling in oxide superlattices. Nat. Mater. 11, 675-681 (2012).

15. Lin, M.-L. et al. Cross-dimensional electron-phonon coupling in van der Waals heterostructures. Nat. Commun. 10, 2419 (2019).

16. Leroux, M. et al. Strong anharmonicity induces quantum melting of charge density wave in $2 \mathrm{H}$ - $\mathrm{NbSe}_{2}$ under pressure. Phys. Rev. B 92, 140303 (2015).

17. Strocov, V. N. et al. Three-dimensional electron realm in $\mathrm{VSe}_{2}$ by soft-x-ray photoelectron spectroscopy: origin of charge-density waves. Phys. Rev. Lett. 109, 086401 (2012).

18. Tsutsumi, K. X-ray-diffraction study of the periodic lattice distortion associated with a charge-density wave in $1 T-\mathrm{VSe}_{2}$. Phys. Rev. B 26, 5756-5759 (1982).

19. Terashima, K. et al. Charge-density wave transition of $1 T-\mathrm{VSe}_{2}$ studied by angle-resolved photoemission spectroscopy. Phys. Rev. B 68, 155108 (2003).

20. Weber, F. et al. Extended phonon collapse and the origin of the charge-density wave in $2 \mathrm{H}-\mathrm{NbSe}_{2}$. Phys. Rev. Lett. 107, 107403 (2011).

21. Calandra, M., Mazin, I. I. \& Mauri, F. Effect of dimensionality on the chargedensity wave in few-layer $2 \mathrm{H}$-NbSe $\mathrm{Nb}_{2}$. Phys. Rev. B 80, 241108 (2009).

22. Weber, F. et al. Electron-phonon coupling and the soft phonon mode in $\mathrm{TiSe}_{2}$ Phys. Rev. Lett. 107, 266401 (2011).

23. Calandra, M. \& Mauri, F. Charge-density wave and superconducting dome in $\mathrm{TiSe}_{2}$ from electron-phonon interaction. Phys. Rev. Lett. 106, 196406 (2011).

24. Bianco, R., Errea, I., Monacelli, L., Calandra, M. \& Mauri, F. Quantum enhancement of charge density wave in $\mathrm{NbS}_{2}$ in the two-dimensional limit. Nano Lett. 19, 3098-3103 (2019).

25. Zhou, J. S. et al. Anharmonicity and doping melt the charge density wave in single-layer $\mathrm{NbSe}_{2}$. Nano Lett. 20, 4809-4815 (2020).

26. Bianco, R., Monacelli, L., Calandra, M., Mauri, F. \& Errea, I. Weak dimensionality dependence and dominant role of ionic fluctuations in the charge-density-wave transition of $\mathrm{NbSe}_{2}$. Phys. Rev. Lett. 125, 106101 (2020).

27. Zhou, J. S. et al. Theory of the thickness dependence of the charge density wave transition in 1T-TiTe 2 . 2D Materials 7, 045032 (2020).

28. Chen, P. et al. Unique gap structure and symmetry of the charge density wave in single-layer $\mathrm{VSe}_{2}$. Phys. Rev. Lett. 121, 196402 (2018).

29. Baker, G. A., Nickel, B. G. \& Meiron, D. I. Critical indices from perturbation analysis of the Callan-Symanzik equation. Phys. Rev. B 17, 1365-1374 (1978).

30. Girault, S., Moudden, A. H. \& Pouget, J. P. Critical x-ray scattering at the Peierls transition of the blue bronze. Phys. Rev. B 39, 4430-4434 (1989).

31. Hoesch, M., Bosak, A., Chernyshov, D., Berger, H. \& Krisch, M. Giant Kohn anomaly and the phase transition in charge density wave $\mathrm{ZrTe}_{3}$. Phys. Rev. Lett. 102, 086402 (2009).

32. Errea, I., Calandra, M. \& Mauri, F. Anharmonic free energies and phonon dispersions from the stochastic self-consistent harmonic approximation: Application to platinum and palladium hydrides. Phys. Rev. B 89, 064302 (2014).

33. Bianco, R., Errea, I., Paulatto, L., Calandra, M. \& Mauri, F. Second-order structural phase transitions, free energy curvature, and temperature-dependent anharmonic phonons in the self-consistent harmonic approximation: theory and stochastic implementation. Phys. Rev. B 96, 014111 (2017). 
34. Monacelli, L., Errea, I., Calandra, M. \& Mauri, F. Pressure and stress tensor of complex anharmonic crystals within the stochastic self-consistent harmonic approximation. Phys. Rev. B 98, 024106 (2018).

35. Pandey, J. \& Soni, A. Electron-phonon interactions and two-phonon modes associated with charge density wave in single crystalline $1 T-\mathrm{VSe}_{2}$. Phys. Rev. Res. 2, 033118 (2020).

36. $\mathrm{Si}, \mathrm{J}$. G. et al. Origin of the multiple charge density wave order in $1 T-\mathrm{VSe}_{2}$. Phys. Rev. B 101, 235405 (2020).

37. Weber, F. et al. Three-dimensional Fermi surface of $2 \mathrm{H}-\mathrm{NbS}_{2}$ : Implications for the mechanism of charge density waves. Phys. Rev. B 97, 235122 (2018).

38. Ugeda, M. M. et al. Characterization of collective ground states in single-layer $\mathrm{NbSe}_{2}$. Nat. Phys. 12, 92-97 (2016).

39. Bluschke, M. et al. Stabilization of three-dimensional charge order in $\mathrm{YBa}_{2} \mathrm{Cu}_{3} \mathrm{O}_{6+x}$ via epitaxial growth. Nat. Commun. 9, 2978 (2018).

40. Kim, H.-H. et al. Uniaxial pressure control of competing orders in a hightemperature superconductor. Science 362, 1040-1044 (2018).

41. He, Y. et al. Persistent low-energy phonon broadening near the charge-order $q$ vector in the bilayer cuprate $\mathrm{Bi}_{2} \mathrm{Sr}_{2} \mathrm{CaCu}_{2} \mathrm{O}_{8+\delta}$. Phys. Rev. B 98, 035102 (2018).

42. Fumega, A. O. et al. Absence of ferromagnetism in $\mathrm{VSe}_{2}$ caused by its charge density wave phase. J. Phys. Chem. C 123, 27802-27810 (2019).

43. Said, A. H. et al. High-energy-resolution inelastic X-ray scattering spectrometer at beamline 30 -ID of the advanced photon source. J. Synchrotron Radiat. 27, 827-835 (2020).

44. Perdew, J. P., Burke, K. \& Ernzerhof, M. Generalized gradient approximation made simple. Phys. Rev. Lett. 77, 3865-3868 (1996).

45. Grimme, S. Semiempirical GGA-type density functional constructed with a long-range dispersion correction. J. Comput. Chem. 27, 1787-1799 (2006).

46. Baroni, S., de Gironcoli, S., Dal Corso, A. \& Giannozzi, P. Phonons and related crystal properties from density-functional perturbation theory. Rev. Mod. Phys. 73, 515-562 (2001).

47. Giannozzi, P. et al. QUANTUM ESPRESSO: a modular and open-source software project for quantum simulations of materials. J. Phys. Condens. Matter 21, 395502 (2009).

48. Giannozzi, P. et al. Advanced capabilities for materials modelling with QUANTUM ESPRESSO. J. Phys.: Condens. Matter 29, 465901 (2017).

49. Marzari, N. \& Vanderbilt, D. Maximally localized generalized Wannier functions for composite energy bands. Phys. Rev. B 56, 12847-12865 (1997).

50. Souza, I., Marzari, N. \& Vanderbilt, D. Maximally localized Wannier functions for entangled energy bands. Phys. Rev. B 65, 035109 (2001).

51. Mostofi, A. A. et al. Wannier90: a tool for obtaining maximally-localised Wannier functions. Comput. Phys. Commun. 178, 685-699 (2008).

52. Aroyo, M. I. et al. Brillouin-zone database on the Bilbao Crystallographic Server. Acta Crystallogr. A 70, 126-137 (2014).

\section{Acknowledgements}

The authors acknowledge valuable discussions with V. Pardo, A. O. Fumega and M. Hoesch. S.B-C thanks the MINECO of Spain through the project PGC2018-101334-AC22. F.M. and L.M. acknowledge support by the MIUR PRIN-2017 program, project number 2017Z8TS5B. M.C. acknowledges support from Agence Nationale de la Recherche, Project ACCEPT, Grant No. ANR-19-CE24-0028 and M.C and F.M. the
Graphene Flagship Core 3. Calculations were performed at the Joliot Curie-AMD supercomputer under the PRACE project RA4956. This research used resources of the Advanced Photon Source, a U.S. Department of Energy (DOE) Office of Science User Facility, operated for the DOE Office of Science by Argonne National Laboratory under Contract No. DE-AC02-06CH11357. Extraordinary facility operations were supported in part by the DOE Office of Science through the National Virtual Biotechnology Laboratory, a consortium of DOE national laboratories focused on the response to COVID-19, with funding provided by the Coronavirus CARES Act.

\section{Author contributions}

S.B.-C. conceived and managed the project. S.K.M. and K.R. synthesized and S.B.-C. characterized the samples. A.H.S. and S.B.-C. carried out the high-resolution IXS experiments. S.B.-C. analyzed the experimental data. J.D., R.B., L.M., M.C., F.M. and I.E performed the first principles calculations. S.B.-C. and I.E. wrote the manuscript with input from all co-authors.

\section{Competing interests}

The authors declare no competing interests.

\section{Additional information}

Supplementary information is available for this paper at https://doi.org/10.1038/s41467 020-20829-2.

Correspondence and requests for materials should be addressed to I.E. or S.B.-C.

Peer review information Nature Communications thanks Ajay Soni and the other anonymous, reviewer(s) for their contribution to the peer review of this work. Peer reviewer reports are available.

Reprints and permission information is available at http://www.nature.com/reprints

Publisher's note Springer Nature remains neutral with regard to jurisdictional claims in published maps and institutional affiliations.

\begin{abstract}
(c) (i) Open Access This article is licensed under a Creative Commons BY Attribution 4.0 International License, which permits use, sharing, adaptation, distribution and reproduction in any medium or format, as long as you give appropriate credit to the original author(s) and the source, provide a link to the Creative Commons license, and indicate if changes were made. The images or other third party material in this article are included in the article's Creative Commons license, unless indicated otherwise in a credit line to the material. If material is not included in the article's Creative Commons license and your intended use is not permitted by statutory regulation or exceeds the permitted use, you will need to obtain permission directly from the copyright holder. To view a copy of this license, visit http://creativecommons.org/ licenses/by/4.0/.
\end{abstract}

(C) The Author(s) 2021 